\title{
SYNTHESIS, CHARACTERIZATION AND BIOLOGICAL EVALUATION OF SUBSTITUTED 1,3,4-OXADIAZOLE DERIVATIVE: DERIVED FROM CIPROFLOXACIN
}

\begin{abstract}
ANUJ SINGHAI ${ }^{1 *}$, M. K. GUPTA ${ }^{2}$
${ }^{1}$ Department of Pharmaceutical Chemistry, Oriental College of Pharmacy and Research, Oriental University, Indore, Madhya Pradesh, India. ${ }^{2}$ Department of Pharmacognosy, Oriental College of Pharmacy and Research, Oriental University, Indore, Madhya Pradesh, India. Email: anujsinghai1989@gmail.com
\end{abstract}

Received: 27 June 2019, Revised and Accepted: 23 July 2019

ABSTRACT

Objective: The purpose of this research is synthesized and evaluates different derivatives of oxadiazole.

Methods: A novel series of substituted 1,3,4-oxadiazole derivative were synthesized by condensing different amine with 1-cyclopropyl-6-fluoro-7(piperazin-1-yl)-3-(5-thioxo-4,5-dihydro-1,3,4-oxadiazol-2-yl)quinolin-4(1H)-one (III) in the presence of formaldehyde. The structure of these novel synthesized compounds was characterized on the bases of physicochemical and spectral analysis. The title compounds (IVa-h) were screened for antibacterial activity by disc diffusion method.

Results: Substituted 1,3,4-oxadiazole derivative was synthesized, characterized, and evaluated for antibacterial activity. Compounds IVa, IVd, IVe, IVf, and IV h showed enhance activities then ciprofloxacin against all Gram-positive and Gram-negative organisms. Compound IVe showed the highest activity against Staphylococcus aureus and compound IV showed the highest activity against Escherichia coli.

Conclusion: The present study demonstrates the synthesis and characterization of 1,3,4-oxadiazole derivatives derived from ciprofloxacin. These compounds were evaluated for antibacterial activity against different Gram-positive and Gram-negative organism. In some cases, antibacterial activity is found to be enhanced as compared to standard drug ciprofloxacin.

Keywords: 1,3,4-Oxadiazole, Ciprofloxacin, Antimicrobial.

(C) 2019 The Authors. Published by Innovare Academic Sciences Pvt Ltd. This is an open access article under the CC BY license (http://creativecommons. org/licenses/by/4. 0/) DOI: http://dx.doi.org/10.22159/ajpcr.2019.v12i9.34712

\section{INTRODUCTION}

Chemical modification of bioactive components is one of the most common approaches in drug discovery and development with an improved therapeutic effect. As resistance to antimicrobial drugs is widespread, there is an increasing need for the identification of novel structure leads that may be of use in designing new, potent, and less toxic antimicrobial agents.

1,3,4-Oxadiazole is an important class of heterocyclic compounds containing one oxygen and two nitrogen atoms in five-member ring with a broad spectrum of biological activities [1,2]. During the past year, considerable evidence have accumulated to demonstrate the efficiency of 1,3,4-oxadiazole including antimicrobial [3,4], antifungal [5], anti-HIV [6], anthelmintic [7], anticancer [2], anticonvulsant [8], antiviral [9], antimalarial [10], hypoglycemic [11], anti-inflammatory [12], analgesic [13], antitubercular [14], and other biological properties such as genotoxic studies and lipid peroxidation inhibitor [15].

In this paper, we have focused on the incorporation of 1,3,4-oxadiazole with ciprofloxacin in one framework. Oxadiazole ring was introduced to the carboxylic side chain and different amines were attached to oxadiazole. In some cases, antibacterial activity is found to be enhanced as compared to standard drug ciprofloxacin.

Ciprofloxacin is the first widely used quinolone with advanced systemic activity, marketed in 1987. The second-generation antibiotics, now called fluoroquinolones, have excellent activity against many Gram-negative bacteria. Fluoroquinolones constituted a significant advancement in the management of infectious diseases [16]. Ciprofloxacin is used for the treatment of urinary tract infection [17], prostatitis [18], continuous ambulatory peritoneal dialysis infection [19], antitumor activity [20], etc.
Ciprofloxacin is found to be an important antibacterial agent. Keeping this in view, it was thought worthwhile to design the synthesis of title compounds, wherein the biological activity of ciprofloxacin is enhanced by 1,3,4-oxadiazole.

\section{METHODS}

Chemicals used in this synthetic work were purchased from S.D. Fine-Chem Ltd., Mumbai, and Sigma-Aldrich, India (Merck). Solvents except laboratory reagent grade were dried and purified according to the literature when necessary. The purity of the compounds was checked on thin-layer chromatography (TLC) plates using silica gel G as stationary phase and iodine vapors as a visualizing agent. Melting points of synthesized compounds were determined using Thermonik melting point apparatus and are uncorrected; IR spectra were recorded on Thermo Nicolet Spectrophotometer using $\mathrm{KBr}$ pellets. The proton nuclear magnetic resonance $\left({ }^{1} \mathrm{H}\right.$ NMR) was recorded on Bruker Avance II NMR $500 \mathrm{MHz}$ instruments using appropriated solvent and TMS as internal standard, chemical shifts are expressed as $\delta$ values (ppm).

\section{Synthesis and spectral studies}

The title compounds were synthesized as given in Scheme 1.

\section{Synthesis of methyl ester of ciprofloxacin (I)}

The methyl ester was prepared as per procedure reported in literature [21]. M.p. $244-246^{\circ} \mathrm{C}$, yield: $78.43 \%$. IR spectra showed bands at $3088(\mathrm{C}-\mathrm{H}), 1745(\mathrm{C}=\mathrm{O}), 1531(\mathrm{C}=\mathrm{C}), 1476(\mathrm{C}-\mathrm{N})$, and 1330 (C-F). ${ }^{1} \mathrm{H}$ NMR chemical shift at $\left(\mathrm{CDCl}_{3}, \delta \mathrm{ppm}\right) 8.45\left(\mathrm{~s}, 1 \mathrm{H}, 2^{\text {nd }}\right.$ aryl $\left.\mathrm{H}\right)$, $8.05\left(\mathrm{~d}, 1 \mathrm{H}, 5^{\text {th }}\right.$ aryl $\left.\mathrm{H}\right), 6.84\left(\mathrm{~s}, 1 \mathrm{H}, 8^{\text {th }}\right.$ aryl $\left.\mathrm{H}\right), 3.78\left(\mathrm{~s}, 3 \mathrm{H}, \mathrm{CH}_{3}\right), 3.76-$ 2.32 (m, 9H, piperznyl $\mathrm{H}$ ), 2.55 ( $\mathrm{m}, 1 \mathrm{H}$ of cyclopropane), and 1.78-1.44 (m, $4 \mathrm{H}$ of cyclopropane). 
<smiles>COC(=O)c1cn(C2CC2)c2cc(N3CCNCC3)c(F)cc2c1=O</smiles><smiles>[R]Cn1nc(-c2cn(C3CC3)c3cc(N4CCNCC4)c(F)cc3c2=O)oc1=S</smiles>

IVa-h<smiles>O=c1c(-c2n[nH]c(=S)o2)cn(C2CC2)c2cc(N3CCNCC3)c(F)cc12</smiles>

Scheme 1: a- CH3OH/ H2SO4, b- CH3OH/ NH2NH2H2O, c- CH3OH/CS2 / KOH, d- CH3OH/HCHO/ Amine, R- Different amines Synthesis of 1,3,4-oxadiazole derivatives

Table 1: Physicochemical data of the different synthesized title compounds (IVa-h)

\begin{tabular}{|c|c|c|c|c|c|}
\hline Compound & $\mathbf{R}$ & M.P. $\left({ }^{\circ} \mathrm{C}\right)$ & Yield (\%) & Rf* & Molecular formula \\
\hline IVa & & $232-234$ & 74.5 & 0.30 & $\mathrm{C}_{23} \mathrm{H}_{27} \mathrm{FN}_{6} \mathrm{O}_{3} \mathrm{~S}$ \\
\hline $\mathrm{IVb}$ & & $216-218$ & 69.9 & 0.41 & $\mathrm{C}_{24} \mathrm{H}_{29} \mathrm{FN}_{6} \mathrm{O}_{2} \mathrm{~S}$ \\
\hline IVc & & $202-204$ & 70.7 & 0.29 & $\mathrm{C}_{25} \mathrm{H}_{31} \mathrm{FN}_{6} \mathrm{O}_{2} \mathrm{~S}$ \\
\hline IVd & & $184-186$ & 70.7 & 0.44 & $\mathrm{C}_{23} \mathrm{H}_{28} \mathrm{FN}_{7} \mathrm{O}_{2} \mathrm{~S}$ \\
\hline IVe & & $194-196$ & 71.4 & 0.53 & $\mathrm{C}_{24} \mathrm{H}_{30} \mathrm{FN}_{7} \mathrm{O}_{2} \mathrm{~S}$ \\
\hline IVf & & $215-217$ & 68.2 & 0.24 & $\mathrm{C}_{23} \mathrm{H}_{29} \mathrm{FN}_{6} \mathrm{O}_{2} \mathrm{~S}$ \\
\hline IVg & & $242-244$ & 75.5 & 0.43 & $\mathrm{C}_{31} \mathrm{H}_{41} \mathrm{FN}_{6} \mathrm{O}_{2} \mathrm{~S}$ \\
\hline IVh & & $178-180$ & 72.8 & 0.64 & $\mathrm{C}_{25} \mathrm{H}_{31} \mathrm{FN}_{6} \mathrm{O}_{2} \mathrm{~S}$ \\
\hline
\end{tabular}

All compounds were recrystallized by methanol. Stationary phase* - silica gel G. Mobile phase - ethyl acetate: chloroform (4:1). Visualizing agent - iodine vapors

Synthesis of 1-cyclopropyl-6-fluoro-4-oxo-7-(piperazin-1-yl)-1,4dihydroquinoline-3-carbohydrazide (II)

Compound I (0.01 mol) and hydrazine hydrate $(99 \%)(0.02 \mathrm{~mol})$ were refluxed in absolute methanol $(50 \mathrm{ml})$ for $20 \mathrm{~h}$ (monitored by TLC). The mixture was concentrated, cooled, and poured in ice-cold water. The solid thus separated, filtered, dried, and recrystallized from ethanol:water (4:1). M.p. $256-258^{\circ} \mathrm{C}$, yield: $75 \%$. IR spectra showed bands at $\mathrm{N}-\mathrm{H}$ at $3325 \mathrm{~cm}^{-1} 3058(\mathrm{C}-\mathrm{H}), 1645$ (C=0), 1511 (C=C), 1466 $(\mathrm{C}-\mathrm{N})$, and $1320(\mathrm{C}-\mathrm{F}) .{ }^{1} \mathrm{H}$ NMR chemical shift at $\left(\mathrm{CDCl}_{3}, \delta \mathrm{ppm}\right) 8.96(\mathrm{~s}$, $1 \mathrm{H}, 2^{\text {nd }}$ aryl $\left.\mathrm{H}\right), 8.15\left(\mathrm{~d}, 1 \mathrm{H}, 5^{\text {th }}\right.$ aryl $\left.\mathrm{H}\right), 6.14\left(\mathrm{~s}, 1 \mathrm{H}, 8^{\text {th }}\right.$ aryl $\left.\mathrm{H}\right), 3.66-2.12$ (m, 9H, piperazinyl H), 2.65 (m, 1H of cyclopropane), 1.88-1.44 (m, 4H of cyclopropane), and $7.90(\mathrm{~s}, 1 \mathrm{H}, \mathrm{NH}), 2.15\left(\mathrm{~s}, 2 \mathrm{H}, \mathrm{NH}_{2}\right)$.

Synthesis of 1-cyclopropyl-6-fluoro-7-(piperazin-1-yl)-3-(5thioxo-4,5-dihydro-1,3,4-oxadiazol-2-yl)quinolin-4(1H)-one (III) A mixture of II $(0.005 \mathrm{~mol}) \mathrm{KOH}(0.005 \mathrm{~mol})$ and carbon disulfide $(5 \mathrm{ml})$ in methanol $(50 \mathrm{ml})$ were refluxed on a steam bath for $12 \mathrm{~h}$ (monitored by TLC). The solution was then concentrated, cooled, and acidified with dil. $\mathrm{HCl}$. The solid mass that separated, filtered, washed with ethanol, dried, and recrystallized from ethanol:water $(4: 1)$. Mp $238-240^{\circ} \mathrm{C}$, 
Table 2: Zone of inhibition (mm) of ciprofloxacin and their derivatives against various microorganism

\begin{tabular}{|c|c|c|c|c|c|c|c|c|c|}
\hline Organism & Ciprofloxacin $(50 \mathrm{ug} / \mathrm{ml})$ & IVa & IVb & IVc & IVd & IVe & IVf & IVg & IVh \\
\hline Staphylococcus aureus & 24 & 26 & 22 & 23 & 26 & 28 & 25 & 21 & 27 \\
\hline Bacillus subtilis & 18 & 22 & 16 & 16 & 21 & 25 & 19 & 17 & 23 \\
\hline Staphylococcus pneumoniae & 19 & 20 & 18 & 19 & 24 & 22 & 19 & 17 & 21 \\
\hline Escherichia coli & 21 & 28 & 22 & 20 & 24 & 24 & 22 & 16 & 22 \\
\hline Pseudomonas aeruginosa & 22 & 23 & 21 & 20 & 26 & 27 & 23 & 19 & 24 \\
\hline Klebsiella pneumonia & 16 & 15 & 12 & 15 & 18 & 18 & 15 & 15 & 18 \\
\hline Salmonella typhi & 15 & 14 & 11 & 10 & 16 & 16 & 16 & 15 & 17 \\
\hline
\end{tabular}

yield: $72 \%$. IR spectra showed bands at N-H at $3373 \mathrm{~cm}^{-1} 3068(\mathrm{C}-\mathrm{H})$, $1576(\mathrm{C}=\mathrm{N}), 1511(\mathrm{C}=\mathrm{C}), 1456(\mathrm{C}-\mathrm{N}), 1312(\mathrm{C}=\mathrm{S}), 1300(\mathrm{C}-\mathrm{F})$, and 1249 (C-O-C). ${ }^{1} \mathrm{H}$ NMR chemical shift at $\left(\mathrm{CDCl}_{3}, \delta \mathrm{ppm}\right) 8.90(\mathrm{~s}, 1 \mathrm{H}, \mathrm{NH}), 8.66$ $\left(\mathrm{s}, 1 \mathrm{H}, 2^{\text {nd }}\right.$ aryl $\left.\mathrm{H}\right), 8.35\left(\mathrm{~d}, 1 \mathrm{H}, 5^{\text {th }}\right.$ aryl $\left.\mathrm{H}\right), 6.54\left(\mathrm{~s}, 1 \mathrm{H}, 8^{\text {th }}\right.$ aryl $\left.\mathrm{H}\right), 3.16-$ 2.02 ( $\mathrm{m}, 9 \mathrm{H}$, piperznyl $\mathrm{H}$ ), 3.65 ( $\mathrm{m}, 1 \mathrm{H}$ of cyclopropane), and 1.78-1.34 (m, $4 \mathrm{H}$ of cyclopropane).

\section{General procedure for the synthesis of derivatives (IVa-h)}

To a solution of III $(0.01 \mathrm{~mol})$ in ethanol, a mixture of formaldehyde $(0.015 \mathrm{~mol})$ and a secondary amine $(0.01 \mathrm{~mol})$ in ethanol was added with stirring. After complete addition, the stirring was continued overnight at room temperature. The precipitated solids were filtered, washed with water, and recrystallized from methanol.

1-cyclopropyl-6-fluoro-3-(4-(morpholinomethyl)-5-thioxo-4,5dihydro-1,3,4-oxadiazol-2yl)-7-(piperazin-1yl)quinolin-4(1H)one (IVa)

This was obtained by reacting 1-cyclopropyl-6-fluoro-7-(piperazin-1yl)-3-(5-thioxo-4,5-dihydro-1,3,4-oxadiazol-2-yl) quinolin-4(1H)-one (III, $0.01 \mathrm{~mol})$ and morpholine $(0.015 \mathrm{~mol})$ as described in general procedure. Mp $232-234^{\circ} \mathrm{C}$, yield: $74.5 \%$. IR spectra showed bands at 3068 (C-H), 1576 (C=N), 1511 (C=C), 1456 (C-N), 1312 (C=S), 1300 (CF), and 1249 (C-O-C). ${ }^{1} \mathrm{H}$ NMR chemical shift at $\left(\mathrm{CDCl}_{3}, \delta \mathrm{ppm}\right) 8.14(\mathrm{~s}, 1 \mathrm{H}$, $2^{\text {nd }}$ aryl $\left.\mathrm{H}\right), 7.95\left(\mathrm{~d}, 1 \mathrm{H}, 5^{\text {th }}\right.$ aryl $\left.\mathrm{H}\right), 6.14\left(\mathrm{~s}, 1 \mathrm{H}, 8^{\text {th }}\right.$ aryl $\left.\mathrm{H}\right), 3.46-2.10(\mathrm{~m}$, 9H, piperznyl $\mathrm{H}), 3.72\left(\mathrm{~s}, 2 \mathrm{H}, \mathrm{N}-\mathrm{CH}_{2}-\mathrm{N}\right), 3.68-3.65(\mathrm{t}, 4 \mathrm{H}$, morpholine), 2.79-2.76 (t, 4H, morpholine), 2.65 (m,1H of cyclopropane), and 1.881.44 (m, $4 \mathrm{H}$ of cyclopropane)

1-cyclopropyl-6-fluoro-7-(piperazin-1yl)-3-(4-(piperidin-1ylmethyl)-5-thioxo-4,5 dihydro-1,3,4-oxadiazol-2yl)-quinolin4(1H)-one (IVb)

This was obtained by reacting 1-cyclopropyl-6-fluoro-7-(piperazin1-yl)-3-(5-thioxo-4,5-dihydro-1,3,4-oxadiazol-2-yl)quinolin-4(1H)one (III, $0.01 \mathrm{~mol})$ and piperidine $(0.015 \mathrm{~mol})$ as described in general procedure. $\mathrm{Mp} 216-218^{\circ} \mathrm{C}$, yield: $69.6 \%$. IR spectra showed bands at 3075 (C-H), 1540 (C=N), 1525 (C=C), 1460 (C-N), 1320 (C=S), 1310 (CF), and $1230(\mathrm{C}-0-\mathrm{C}) .{ }^{1} \mathrm{H}$ NMR chemical shift at $\left(\mathrm{CDCl}_{3}, \delta \mathrm{ppm}\right) 8.24(\mathrm{~s}$, $1 \mathrm{H}, 2^{\text {nd }}$ aryl $\left.\mathrm{H}\right), 7.85\left(\mathrm{~d}, 1 \mathrm{H}, 5^{\text {th }}\right.$ aryl $\left.\mathrm{H}\right), 6.18\left(\mathrm{~s}, 1 \mathrm{H}, 8^{\text {th }}\right.$ aryl H), 3.66-2.20 $(\mathrm{m}, 9 \mathrm{H}$, piperznyl $\mathrm{H}), 3.42\left(\mathrm{~s}, 2 \mathrm{H}, \mathrm{N}-\mathrm{CH}_{2}-\mathrm{N}\right), 2.80-2.72(\mathrm{t}, 4 \mathrm{H}$, piperidine), $1.64(\mathrm{~m}, 2 \mathrm{H}$, piperidine), $1.51-1.24$ (m, 4H, piperidine), 2.55 (m, $1 \mathrm{H}$ of cyclopropane), and 1.28-1.04 (m, $4 \mathrm{H}$ of cyclopropane).

1-cyclopropyl-6-fluoro-3-(4-((2-methylpiperidin-1-ylmethyl)5-thioxo-4,5-dihydro-1,3,4-oxadiazol-2yl)-7-(piperazin-1yl) quinolin-4(1H)-one (IVc)

This was obtained by reacting 1-cyclopropyl-6-fluoro-7-(piperazin-1yl)-3-(5-thioxo-4,5-dihydro-1,3,4-oxadiazol-2-yl) quinolin-4(1H)-one (III, $0.01 \mathrm{~mol})$ and 2-methyl piperidine $(0.015 \mathrm{~mol})$ as described in general procedure. $\mathrm{Mp} 202-204^{\circ} \mathrm{C}$, yield: $70.7 \%$. IR spectra showed bands at $3078(\mathrm{C}-\mathrm{H}), 1563(\mathrm{C}=\mathrm{N}), 1512(\mathrm{C}=\mathrm{C}), 1474(\mathrm{C}-\mathrm{N}), 1355(\mathrm{C}=\mathrm{S})$, 1313 (C-F), and 1263 (C-O-C). ${ }^{1} \mathrm{H}$ NMR chemical shift at ( $\left.\mathrm{CDCl}_{3}, \delta \mathrm{ppm}\right)$ $8.04\left(\mathrm{~s}, 1 \mathrm{H}, 2^{\text {nd }}\right.$ aryl $\left.\mathrm{H}\right), 7.65\left(\mathrm{~d}, 1 \mathrm{H}, 5^{\text {th }}\right.$ aryl $\left.\mathrm{H}\right), 6.66\left(\mathrm{~s}, 1 \mathrm{H}, 8^{\text {th }}\right.$ aryl $\left.\mathrm{H}\right)$, 3.16-2.16 (m, 9H, piperznyl H), $3.92\left(\mathrm{~s}, 2 \mathrm{H}, \mathrm{N}-\mathrm{CH}_{2}-\mathrm{N}\right), 2.80-2.72(\mathrm{~m}, 1 \mathrm{H}$, piperidine), 1.74-1.55 (m, 6H, piperidine), 1.37-1.35 (d, 3H, 2-methyl piperidine), 1.25 ( $\mathrm{m}, 2 \mathrm{H}$, piperidine), 2.51 ( $\mathrm{m}, 1 \mathrm{H}$ of cyclopropane), and 1.26-1.08 (m, 4H of cyclopropane). 1-cyclopropyl-6-fluoro-7-(piperazin-1-yl)-3-(4-(piperazin-1ylmethyl)-5-thioxo-4,5-dihydro-1,3,4-oxadiazol-2-yl)quinolin4(1H)-one (IVd)

This was obtained by reacting 1-cyclopropyl-6-fluoro-7-(piperazin1-yl)-3-(5-thioxo-4,5-dihydro-1,3,4-oxadiazol-2-yl) quinolin-4(1H)one (III, $0.01 \mathrm{~mol})$ and piperazine $(0.015 \mathrm{~mol})$ as described in general procedure. Mp $184-186^{\circ} \mathrm{C}$, yield: $70.7 \%$. IR spectra showed bands at $3080(\mathrm{C}-\mathrm{H}), 1566(\mathrm{C}=\mathrm{N}), 1520(\mathrm{C}=\mathrm{C}), 1466(\mathrm{C}-\mathrm{N}), 1322$ (C=S), 1310 (C-F), and 1239 (C-O-C). ${ }^{1} \mathrm{H}$ NMR chemical shift at $\left(\mathrm{CDCl}_{3}, \delta \mathrm{ppm}\right) 8.17\left(\mathrm{~s}, 1 \mathrm{H}, 2^{\text {nd }}\right.$ aryl $\left.\mathrm{H}\right), 7.45\left(\mathrm{~d}, 1 \mathrm{H}, 5^{\text {th }}\right.$ aryl $\left.\mathrm{H}\right), 6.75(\mathrm{~s}$, $1 \mathrm{H}, 8^{\text {th }}$ aryl $\left.\mathrm{H}\right), 3.66-2.36(\mathrm{~m}, 9 \mathrm{H}$, piperznyl $\mathrm{H}), 3.52\left(\mathrm{~s}, 2 \mathrm{H}, \mathrm{N}-\mathrm{CH}_{2}-\mathrm{N}\right)$, 2.62-2.47 (t, 4H, piperazine), 2.39-2.32 (t, $4 \mathrm{H}$, piperazine), $1.91(\mathrm{~m}$, $1 \mathrm{H}$, piperazine), 2.51 ( $\mathrm{m}, 1 \mathrm{H}$ of cyclopropane), and 1.26-1.08 (m, $4 \mathrm{H}$ of cyclopropane).

1-cyclopropyl-6-fluoro-3-(4-((4-methylpiperazin-1-yl)methyl)5-thioxo-4,5-dihydro-1,3,4 -oxadiazol-2-yl)-7-(piperazin-1-yl) quinolin-4(1H)-one (IVe)

This was obtained by reacting 1-cyclopropyl-6-fluoro-7-(piperazin-1yl)-3-(5-thioxo-4,5-dihydro-1,3,4-oxadiazol-2-yl)quinolin-4(1H)-one (III, $0.01 \mathrm{~mol})$ and $\mathrm{N}$-methylpiperazine $(0.015 \mathrm{~mol})$ as described in general procedure. Mp $194-196^{\circ} \mathrm{C}$, yield: $71.4 \%$. IR spectra showed bands at $3065(\mathrm{C}-\mathrm{H}), 1545(\mathrm{C}=\mathrm{N}), 1521(\mathrm{C}=\mathrm{C}), 1446(\mathrm{C}-\mathrm{N}), 1332(\mathrm{C}=\mathrm{S})$, 1314 (C-F), and 1239 (C-O-C). ${ }^{1} \mathrm{H}$ NMR chemical shift at ( $\left.\mathrm{CDCl}_{3}, \delta \mathrm{ppm}\right)$ $8.17\left(\mathrm{~s}, 1 \mathrm{H}, 2^{\text {nd }}\right.$ aryl $\left.\mathrm{H}\right), 7.85\left(\mathrm{~d}, 1 \mathrm{H}, 5^{\text {th }}\right.$ aryl $\left.\mathrm{H}\right), 6.15\left(\mathrm{~s}, 1 \mathrm{H}, 8^{\text {th }}\right.$ aryl $\left.\mathrm{H}\right)$, 3.26-2.56 (m, 9H, piperznyl H), $3.02\left(\mathrm{~s}, 2 \mathrm{H}, \mathrm{N}-\mathrm{CH}_{2}-\mathrm{N}\right), 2.52-2.37(\mathrm{t}, 8 \mathrm{H}$, piperazine), $2.23\left(\mathrm{~s}, 3 \mathrm{H}, \mathrm{CH}_{3}\right), 2.51$ ( $\mathrm{m}, 1 \mathrm{H}$ of cyclopropane), and 1.261.08 (m, 4H of cyclopropane).

1-cyclopropyl-3-(4-((diethyl amino)methyl)-5-thioxo-4,5-dihydro1,3,4-oxadiazol-2-yl)6-fluoro-7-(piperazin-1-yl)quinolin-4(1H)one (IVf)

This was obtained by reacting 1-cyclopropyl-6-fluoro-7-(piperazin-1yl)-3-(5-thioxo-4,5-dihydro-1,3,4-oxadiazol-2-yl) quinolin-4(1H)-one (III, $0.01 \mathrm{~mol})$ and diethyl amine $(0.015 \mathrm{~mol})$ as described in general procedure. Mp $215-217^{\circ} \mathrm{C}$, yield: $68.20 \%$. IR spectra showed bands at 3045 (C-H), 1546 (C=N), 1530 (C=C), 1470 (C-N), 1325 (C=S), 1322 (C$\mathrm{F})$, and $1242(\mathrm{C}-\mathrm{O}-\mathrm{C}) .{ }^{1} \mathrm{H}$ NMR chemical shift at $\left(\mathrm{CDCl}_{3}, \delta \mathrm{ppm}\right) 8.02(\mathrm{~s}$, $1 \mathrm{H}, 2^{\text {nd }}$ aryl $\left.\mathrm{H}\right), 7.55\left(\mathrm{~d}, 1 \mathrm{H}, 5^{\text {th }}\right.$ aryl $\left.\mathrm{H}\right), 6.05\left(\mathrm{~s}, 1 \mathrm{H}, 8^{\text {th }}\right.$ aryl $\left.\mathrm{H}\right), 3.16-2.36$ (m, 9H, piperznyl H), $3.08\left(\mathrm{~s}, 2 \mathrm{H}, \mathrm{N}-\mathrm{CH}_{2}-\mathrm{N}\right), 3.06-2.70\left(\mathrm{~m}, 4 \mathrm{H}, \mathrm{CH}_{2}, \mathrm{CH}_{2}\right)$, 1.49-1.08 (t, $\left.6 \mathrm{H}, \mathrm{CH}_{3}, \mathrm{CH}_{3}\right), 2.61$ (m, $1 \mathrm{H}$ of cyclopropane), and 1.16-1.06 ( $\mathrm{m}, 4 \mathrm{H}$ of cyclopropane).

1-cyclopropyl-3-(4-((dicyclohrxylamino)methyl)-5-thioxo-4,5dihydro-1,3,4-oxadiazol-2-yl)6-fluoro-7-(piperazin-1-yl)quinolin4(1H)-one (IVg)

This was obtained by reacting 1-cyclopropyl-6-fluoro-7-(piperazin-1yl)-3-(5-thioxo-4,5-dihydro-1,3,4-oxadiazol-2-yl) quinolin-4(1H)-one (III, $0.01 \mathrm{~mol})$ and diphenyl amine $(0.015 \mathrm{~mol})$ as described in general procedure. Mp $242-244^{\circ} \mathrm{C}$, yield: $75.50 \%$. IR spectra showed bands 3075 (C-H), 1570 (C=N), 1520 (C=C), 1470 (C-N), 1305 (C=S), 1290 (C$\mathrm{F})$, and $1244(\mathrm{C}-\mathrm{O}-\mathrm{C}) .{ }^{1} \mathrm{H}$ NMR chemical shift at $\left(\mathrm{CDCl}_{3}, \delta \mathrm{ppm}\right) 7.82(\mathrm{~s}$, $1 \mathrm{H}, 2^{\text {nd }}$ aryl $\left.\mathrm{H}\right), 7.25\left(\mathrm{~d}, 1 \mathrm{H}, 5^{\text {th }}\right.$ aryl $\left.\mathrm{H}\right), 6.16\left(\mathrm{~s}, 1 \mathrm{H}, 8^{\text {th }}\right.$ aryl $\left.\mathrm{H}\right), 7.12-6.95$ (m, 10H of diphenyl amine) 3.76-2.66 (m, 9H, piperznyl $\mathrm{H}$ ), 3.58 (s, $\left.2 \mathrm{H}, \mathrm{N}-\mathrm{CH}_{2}-\mathrm{N}\right), 2.61(\mathrm{~m}, 1 \mathrm{H}$ of cyclopropane), and 1.16-1.06 (m, $4 \mathrm{H}$ of cyclopropane). 
3-(4((cyclohexylamino)methyl)-5-thioxo-4,5-dihydro-1,3,4oxadiazol-2-yl)-1-cyclopropyl-6-fluoro-7-(piperazin-1-yl) quinolin-4(1H)-one (IVh)

This was obtained by reacting 1-cyclopropyl-6-fluoro-7-(piperazin1-yl)-3-(5-thioxo-4,5-dihydro-1,3,4-oxadiazol-2-yl) quinolin-4(1H)one (III, $0.01 \mathrm{~mol})$ and cyclohexyl amine $(0.015 \mathrm{~mol})$ as described in general procedure. $\mathrm{Mp} 178-180^{\circ} \mathrm{C}$, yield: $72.80 \%$. IR spectra showed bands $3090(\mathrm{C}-\mathrm{H}), 1580(\mathrm{C}=\mathrm{N}), 1536(\mathrm{C}=\mathrm{C}), 1476(\mathrm{C}-\mathrm{N}), 1322(\mathrm{C}=\mathrm{S})$, 1316 (C-F), and 1245 (C-O-C). ${ }^{1} \mathrm{H}$ NMR chemical shift at $\left(\mathrm{CDCl}_{3^{\prime}}, \delta \mathrm{ppm}\right)$ $8.23\left(\mathrm{~s}, 1 \mathrm{H}, 2^{\text {nd }}\right.$ aryl $\left.\mathrm{H}\right), 7.15\left(\mathrm{~d}, 1 \mathrm{H}, 5^{\text {th }}\right.$ aryl $\left.\mathrm{H}\right), 6.55\left(\mathrm{~s}, 1 \mathrm{H}, 8^{\text {th }}\right.$ aryl $\left.\mathrm{H}\right)$, 3.96-2.86 (m, 9H, piperznyl H), $3.75\left(\mathrm{~s}, 2 \mathrm{H}, \mathrm{N}-\mathrm{CH}_{2}-\mathrm{N}\right), 2.57-1.25(\mathrm{~m}$, $11 \mathrm{H}$ cyclohexyl amine), $2.02(\mathrm{~s}, 1 \mathrm{H} \mathrm{NH}) 2.61$ (m, 1H of cyclopropane), and $1.16-1.06(\mathrm{~m}, 4 \mathrm{H}$ of cyclopropane).

Antibacterial activities [22-24]: The antibacterial activity of all newly synthesized derivatives was performed by disc diffusion method. For this activity, $50 \mu \mathrm{g} / \mathrm{mL}$ stock solution of ciprofloxacin and its derivatives were prepared. This method is based on the determination of an inhibited zone proportion to the bacterial susceptibility to the antimicrobial present in the disc.

The result was compared with ciprofloxacin against seven different Gram-positive and Gram-negative organisms, i.e., Staphylococcus aureus, Bacillus subtilis, Staphylococcus pneumonia, Escherichia coli, Pseudomonas aeruginosa, Klebsiella pneumonia, and Salmonella typhi.

\section{RESULTS AND DISCUSSION}

The ciprofloxacin was converted to its methyl esters (I) by esterification. This methyl ester was reacted with hydrazine hydrate, gave carbohydrazide (II). This carbohydrazide was treated with $\mathrm{CS}_{2} /$ $\mathrm{KOH}$ in methanol gave 1-cyclopropyl-6-fluoro-7-(piperazin-1-yl)-3(5-thioxo-4,5-dihydro-1,3,4-oxadiazol-2-yl)quinolin-4(1H)-one) (III). The purity of the compounds was confirmed by melting point; TLC and structure were confirmed by IR and ${ }^{1} \mathrm{H}$ NMR spectral data.

Treatment of 1-cyclopropyl-6-fluoro-7-(piperazin-1-yl)-3-(5-thioxo4,5-dihydro-1,3,4-oxadiazol-2-yl)quinolin-4(1H)-one) (III) with various amines in the presence of formaldehyde gave the title compounds IVa-h. The purity of these compounds was assessed by melting point; TLC and structure were confirmed by IR and ${ }^{1} \mathrm{H}$ NMR. Physicochemical data of the different synthesized title compounds (IVa-h) are given in Table 1.

The result of antibacterial activity is collected in Table 2. The antibacterial activity of all newly synthesized derivatives was performed by disc diffusion method. The result was compared with ciprofloxacin against seven different Gram-positive and Gram-negative organisms and observed that compounds IVa, IVd, IVe, IVf, and IVh showed enhance activities then ciprofloxacin against all Gram-positive and Gramnegative organism. Compound IVg showed less potent activity than ciprofloxacin against all Gram-positive and Gram-negative organisms. Compounds IVb and IVc showed similar activity as ciprofloxacin against Gram-positive and Gram-negative organism. Compound IVe showed the highest activity against $S$. aureus and compound IV showed the highest activity against E. coli. Compounds IVb and IVc showed less active against $S$. typhi. In general, we can say that a total of five derivatives showed enhanced activities out of eight derivatives.

It was observed that when oxadiazole ring was introduced to the carboxylic side chain, significant enhancement of potency against the organism attached to ciprofloxacin.

\section{CONCLUSION}

A series of substituted 1,3,4-oxadiazole were synthesized according to Scheme 1 and the identity of the compounds was confirmed based on their melting point, TLC, IR, and 1H-NMR data. Antibacterial activity was carried out for all the synthesized compounds using disc diffusion method against various Gram-positive and Gram-negative organisms and ciprofloxacin was used as standard. Compounds IVa, IVd, IVe,
IVf, and IV h showed enhance activities then ciprofloxacin against all Gram-positive and Gram-negative organisms. A total of five derivatives showed enhanced activities out of eight derivatives.

\section{ACKNOWLEDGMENT}

We express our thanks to Indian Institute of Science Education and Research, Bhopal, and Indian Institute of Science, Bangalore, for providing spectral analysis of synthesized compounds. We are grateful to Blossom Pharma-Biotech Institute Research Center, Bhopal, for permission to screen the antimicrobial effect of synthesized compounds.

\section{AUTHORS' CONTRIBUTIONS}

Equal.

\section{CONFLICTS OF INTEREST}

None.

\section{REFERENCES}

1. Patel K, Jayachandran E, Shah R, Javali V, Sreenivasa GM. Synthesis, characterization and anthelmintic activity (perituma posthuma) of new oxadiazole incorporated with imidazole and pyrazole. Int J Pharm Bio Sci 2010;1:1-13.

2. Shivarama BH, Poojary KN, Bhat KS, Mithun A, Poojary B. Synthesis and anticancer activity studies on some 2-chloro-1, 4-bis-(5-substituted1,3,4-oxadiazol-2-ylmethyleneoxy) phenylene derivatives. Indian J Chem 2005;44B:1669-73.

3. Chandrakantha B, Shetty $\mathrm{P}$, Nambiyar V, Isloor $\mathrm{N}$, Isloor $\mathrm{AM}$. Synthesis, characterization and biological activity of some new 1,3,4-oxadiazole bearing 2-flouro-4-methoxy phenyl moiety. Eur J Med Chem 2010;45:1206-10.

4. Mistry BD, Desai KR, Intwala SM. Comparative studies of novel oxadiazole derivative having chiral center and their anti-microbial activities. Int J Pharm Pharm Sci 2014;7:195-9.

5. Upadhyay PK, Mishra P. Design, synthesis and antifungal evaluation of novel substituted 1, 3, 4-oxadiazoles, and 1, 3, 4-thiadiazoles. Int J Pharm Pharm Sci 2015;9:467-70.

6. El-Emam AA, Al-Deeb OA, Al-Omara M, Lehmann J. Synthesis, antimicrobial and anti-HIV-1 activity of certain 5-(1-adamantyl)-2substituted thio-1,3,4-oxadiazoles and 5-(1-adamantyl)-3-substituted aminomethyl-1,3,4-oxadiazoline-2-thiones. Bioorg Med Chem 2004;12:5107-13.

7. Manjunath SY, Biradar R, Basawaraj. Synthesis and anthelminitic activity of triheterocycles: [5(5-substituted-3-phenylindol-2-yl)1, 3, 4-oxadiazole-2-yl thiomethyl] benzimidazols. Indian J Heterocycl Chem 2009; 18:321-4.

8. Almasirad A, Tabatabai SA, Faizi M, Kebriaeezadeh A, Mehrabi N, Dalvandi A, et al. Synthesis and anticonvulsant activity of new 2-substituted-5- [2-(2-fluorophenoxy)phenyl]-1,3,4-oxadiazoles and 1,2,4-triazoles. Bioorg Med Chem Lett 2004;14:6057-9.

9. Hassan GS, El-Emam AA, Gad LM, Barghash AE. Synthesis, antimicrobial and antiviral testing of some new 1-adamantyl analogues. Saudi Pharm J 2010;18:123-8.

10. Zareef M, Iqbal R, De Dominguez NG, Rodrigues J, Zaidi JH, Arfan M, et al. Synthesis and antimalarial activity of novel chiral and achiral benzenesulfonamides bearing 1, 3, 4-oxadiazole moieties. J Enzyme Inhib Med Chem 2007;22:301-8.

11. Girges MM. Synthesis and pharmacological evaluation of novel series of sulfonate ester-containing 1,3,4-oxadiazole derivatives with anticipated hypoglycemic activity. Arzneimittelforschung 1994;44:490-5.

12. Palkar MB, Singhai AS, Ronad PM, Vishwanathswamy AH, Boreddy TS, Veerapur VP, et al. Synthesis, pharmacological screening and in silico studies of new class of diclofenac analogues as a promising anti-inflammatory agents. Bioorg Med Chem 2014;22:2855-66.

13. Panda J, Patro VJ, Panda CS, Mishra J. Synthesis, characterization, antibacterial and analgesic evaluation of some 1,3,4-oxadiazole derivatives. Der Pharm Chemic 2011;3:485-90.

14. Siddiqui AA, Islam M, Kumar S. Synthesis and antituberculostic activity of 5-\{3'-oxo-6'(substituted phenyl)-2',3',4',5'-tetrahydropyridazin2'-yl $\}$ methyl-2-substituted 1,3,4-oxadiazole. Der Pharm Lett 2010;2:319-27.

15. Kataria AK, Khan SA, Alam MM, Husain A, Akhtar M, Khanna S, et al. Synthesis of some new 2-(substituted-phenyl)-5-(N,N- 
diphenylaminomethyl)-1,3,4-oxadiazoles: A safer anti-inflammatory and analgesic agents. Acta Pol Pharm 2011;68:381-6.

16. Zhanel GG, Walkty A, Vercaigne L, Karlowsky JA, Embil J, Gin AS, et al. The new fluoroquinolones: A critical review. Can J Infect Dis 1999; 10:207-38

17. Chourasia A, Agrawal S. Development and evaluation of ciprofloxacin hydrochloride loaded ocular insert by using "Plantago ovata" as natural polymer. Int J Curr Pharm Res 2018;10:79-88.

18. Hooper DC, Wolfson JS. Fluoroquinolone antimicrobial agents. N Engl J Med 1991;324:384-94.

19. Ludlam HA, Barton I, White L, McMullin C, King A, Phillips I, et al. Intraperitoneal ciprofloxacin for the treatment of peritonitis in patients receiving continuous ambulatory peritoneal dialysis (CAPD). J Antimicrob Chemother 1990;25:843-51.
20. Yamashita Y, Ashizawa T, Morimoto M, Hosomi J, Nakano H. Antitumor quinolones with mammalian topoisomerase II mediated DNA cleavage activity. Cancer Res 1992;52:2818-22.

21. Furniss B, Hannaford AH, Smith PW, Tachell AR. Vogel's Text Book of Practical Organic Chemistry. $5^{\text {th }}$ ed. London: Prentice Hall; 1998. p. 1077.

22. Robbert DS. Clinical Analysis, Microbiology, Remington's Pharmaceutical Sciences. $18^{\text {th }}$ ed. Peninisilvenia: Mack Publishing Company; 1991. p. 524-7.

23. Tortora GJ, Funke BR, Case CL. Microbiology: An Introduction. $9^{\text {th }}$ ed. San Francisco: Person Education Inc.; 2008. p. 601-2.

24. Pelczar M, Reid R, Cohn E. Antibiotics and other chemotherapeutic agents. In: Microbiology. TMH ed. Noida: TATA-McGraw-Hill Publishing House; 1989. p. 466-93. 